were results of serial surveillance cultures screened for VRE.

The results showed that, of 290 patients enrolled, 35 (12\%) had colonization with VRE on admission. The VRE colonization or infection had been previously detected by clinical cultures in only 4 of these patients. Using logistic regression, VRE colonization at the time of ICU admission was associated with second- and third-generation cephalosporins, length of stay prior to surgical ICU admission, more than one prior ICU stay, and a history of solidorgan transplantation. Eleven $(12.8 \%)$ of 78 patients with follow-up cultures acquired VRE. By pulsed-field gel electrophoresis, two strains predominated, one of which was associated with an overt outbreak on a non-ICU ward near the end of the study period.

It was concluded that VRE colonization was common and usually not recognized by clinical culture. Most patients who had colonization with VRE and were on the surgical ICU acquired VRE prior to surgical ICU entry. Exposure to second- and third-generation cephalosporins, but not vancomycin, was an independent risk factor for colonization. Prospective surveillance of hospitalized patients may yield useful insights about the dissemination of nosocomial VRE beyond what is appreciated by clinical cultures alone.

FROM: Ostrowsky BE, Venkataraman L, D'Agata EM, Gold HS, DeGirolami PC, Samore MH. Vancomycinresistant enterococci in intensive care units: high frequency of stool carriage during a non-outbreak period. Arch Intern Med 1999;159:1467-1472.

\section{Evaluation of Disinfection of Reusable Angioscopes with the Duck Hepatitis B Model}

Transmission of hepatitis B virus (HBV) infection in hospital settings is well known, and there has been a continuing interest to determine the efficacy of disinfection and sterilization procedures on instruments, especially those devices considered difficult to clean. Since HBV has not been cultured, other surrogate hepatitis viruses have been used for studies. Chaufour and coworkers have employed the duck HBV (DHBV), which has similar biological and structural characteristics to HBV and has been adopted as a suitable model for disinfectant testing.

Angioscopic examination of the external jugular vein was performed on DHBV-infected ducks. After use, the instrument was air dried for 3 minutes. Samples were obtained by flushing the channel with $5 \mathrm{~mL}$ of phosphate buffered saline solution. The samples were collected immediately after drying (control); after flushing with $5 \mathrm{~mL}$ of water; after glutaraldehyde disinfection for 5,10 , and 20 minutes; and after ethylene oxide gas sterilization. Angioscopes were either precleaned or uncleaned before disinfection and sterilization. Residual infectivity was assessed with inoculation of samples into the peritoneal cavity of day-old ducks $(\mathrm{n}=231)$.
DNA analysis results of liver samples showed that all 38 control ducks became infected. The frequency of DHBV infection was reduced to $93 \%(14 / 15)$ by flushing the angioscope with $5 \mathrm{~mL}$ of sterile water. No transmission occurred after the use of any of the properly precleaned, disinfected, and sterilized angioscopes. However, after the use of the uncleaned angioscopes, the transmission rate was $90 \%$ $(9 / 10)$ and $70 \%(7 / 10)$ after 5 and 10 minutes of contact time, respectively, in $2 \%$ glutaraldehyde. Even after the recommended 20 minutes of contact time, there was still $6 \%$ $(2 / 35)$ transmission. After ethylene oxide sterilization, two of the recipient ducklings $(2 / 35)$ were infected with DHBV.

It was concluded that there was no disease transmission after reuse of disposable angioscopes adequately cleaned before disinfection or sterilization. However, if the angioscopes are inadequately cleaned, DHBV can survive despite glutaraldehyde disinfection or ethylene oxide sterilization. This contrasts with previous in vitro and in vivo data with solid surgical instruments. It is postulated that the presence of a narrow lumen or residual protein shielding within the lumen may compromise effective inactivation of hepadnaviruses on angioscopes, with the potential risk for patient-to-patient transmission.

FROM: Chaufour X, Deva AK, Vickery K, Zou J, Kumaradeva P, White GH, et al. Evaluation of disinfection and sterilization of reusable angioscopes with the duck hepatitis B model. J Vasc Surg 1999;30:277-282.

\section{Microbial Contamination of Surgical Instruments After Washing}

Surgical instruments exposed to sterile body sites should be contaminated with relatively low levels of microbial contamination or bioburden; however, few studies in the literature have determined the quantitative level and types of contamination. Nancy Chu and colleagues from Advanced Sterilization Products, Johnson \& Johnson, Irvine, CA, performed a study conducted at two clinical sites to determine the level of microbial contamination of surgical instruments after clinical use and after washing. Quantitative assays showed that bioburden levels were in the range of 0 to 4,415 colony-forming units per instrument after clinical use, and $88 \%$ of the instruments had bioburden levels lower than 1,000 .

As expected, a reduction in counts occurred after washing; however, in some cases, higher counts were found on the instruments after the washing process. Although the washing procedure is effective in reducing the microbial levels deposited on the surgical instruments during use, a recontamination process occurs that results in increased counts after washing. The low bioburden level after washing consists of predominantly vegetative microorganisms that present a relatively low challenge to sterilization and disinfection systems.

FROM: Chu NS, Chan-Myers H, Ghazanfari N, Antonoplos P. Levels of naturally occurring microorganisms on surgical instruments after clinical use and after washing. Am J Infect Control 1999;27:315-319. 


\section{Yale University}

Position available; July 1, 2000.

Director of Hospital Epidemiology and co-director of Quality Improvement Support Services (QISS), Yale-New Haven Hospital Yale University School of Medicine.

Seeking an individual at the ASSOCIATE PROFESSOR or PROFESSOR level with excellent skills in hospital epidemiology who also has leadership capabilities and a strong investigative orientation. Primary academic appointment in the Section of Infectious Diseases, Department of Internal Medicine. Individual may desire secondary appointment in the Department of E,pidemiology and Public Health.

The Hospital Epidemiology unit has an integrated staff within QISS, which includes 3 Infection Control Practitioners, a Ql clinical specialist, four epidemiology technicians, a PhD data analyst, and an academic staff (Dr. Louise Dembry, Associate Director and Dr. Robert Baltimore, Associate Hospital Epidemiologist-Pediatrics). Completely equipped and staffed Hospital Epidemiology laboratory. Total budget for program, approaches one million dollars per year. Designec may have responsibility for developing a regional program, with the Yale regionally associated hospitals and clinics. Additional opportunities exist for academic growth.

Yale is an affirmative action/equal opportunity employer. Women and members minority groups are encouraged to apply.

All interested candidates should send their curriculum vitae's to me: Keith Joiner, M.D. Professor and Chief,

Department of Internal Medicine Section of Infectious Diseases Yale University School of Medicine 333 Cedar Street, P.O. Box 208022 Mailcode 108 New Haven, CT 06520-8022

\section{SHEA/SANG-HA Training Course in Infection Control and Hospital Epidemiology}

\author{
November 6-9, 1999 \\ King Fahad National Guard Hospital \\ Riyadh, Saudi Arabia
}

This training program is designed for those who have responsibility for hospital epidemiology and infection control programs. It is sponsored by the Society for Healthcare Epidemiology of America and the Saudia Arabian National Guard Health Affairs (SANG-HA). The course is taught by experts from the United States and the Middle East who support the pursuit of continuing education to broaden the scope of influence through new application of epidemiological skills and continuous improvement of infection control. Topics to be addressed include patterns of transmission of nosocomial infections; risk factors for nosocomial infections; epidemic investigation; review of surveillance techniques; disinfection and sterilization; employee safety and health, and control of bloodborne pathogens; and introduction to quality-improvement challenges facing hospital epidemiologists.
For more information contact: Ziad A. Memish, MD Symposium Chair E-mail: memish@ngha.org Academic Affairs PO Box 22490
Riyadh 11426, Saudi Arabia Fax: 966-1-252-0040
Telephone: $966-1-252-0088$ ext 2329

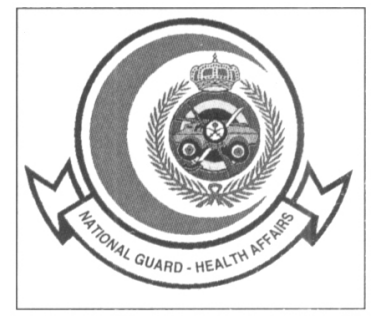

\section{UWHealth University of Wisconsin Hospital and Clinics \\ INFECTION CONTROL SURVEILLANCE PRACTITIONER}

The University of Wisconsin Hospital \& Clinics (UWHC), a leading academic medical center, has a rare opportunity for an experienced Infection Control Practitioner. As part of our Infection Control team, you will work closely with UWHC medical faculty and department heads to implement a cutting-edge infection control program and to develop new patient care protocols and guidelines. You will also have major responsibilities for institution-wide surveillance of nosocomial infections and producing surveillance reports.

To qualify, you must have a Bachelor's degree in an appropriate healthcare field, such as Nursing, Microbiology, Medical Technology, Epidemiology or Preventive Medicine, and at least 5 years of clinical healthcare experience relevant to infection control. Certification in infection control is preferred. If you are the candidate we seek, a competitive salary, an excellent benefits package and great opportunity await you in our top-rated facility and city.

To apply, please send a resume and cover letter to:

University of Wisconsin Hospital and Clinics Human Resources Dept. - Box KS 600 Highland Ave.

Madison, WI 53792

Or Fax at (608) 263-5778

\section{SHEA/ESGNI \\ Training Course in Hospital Epidemiology}

October 29-November 2, 1999

Beach \& Golf Resort Ooghduyne

Julianadorp aan Zee

The Netherlands

This intensive training program is designed for those who have responsibility for hospital epidemiology and infection control programs. It is sponsored by the Society for Healthcare Epidemiology of America and the European Study Group of Nosocomial Infections (ESGNI) of the European Society for Clinical Microbiology and Infectious Diseases (ESCMID). The course is taught by renowned experts from the United States and Europe who are dedicated to continuous quality improvement in infection control and the application of epidemiology within the hospital setting. Topics to be addressed include, but are not limited to, patterns of transmission of nosocomial infections; risk factors for nosocomial infections; approach to epidemic investigation; molecular typing; disinfection and sterilization; epidemic in the OR; introduction to surveillance; use of computerized data in outbreak investigations; MRSA; isolation systems and TB; investigation of excess mortality in the NICU; employee safety and control of bloodborne pathogens; control of antibiotic resistance and antibiotic policies.

For more information, contact University Hospital Nijmegen, 440 MMB, c/o A. Voss, MD, PhD, PO Box 9101, 6500 HB Nijmegen, The Netherlands; fax: 31-24-354-0216; email: y.beeuwkes@mmb.azn.nl. 


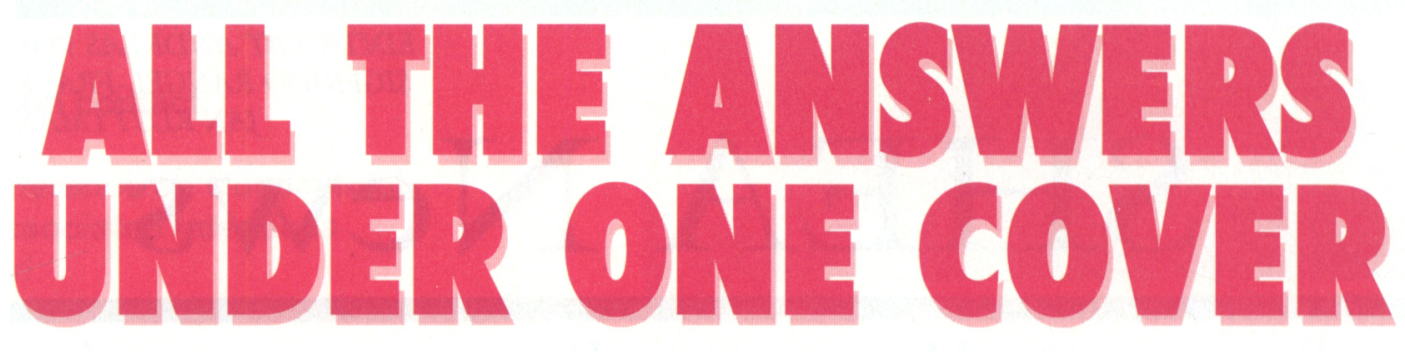

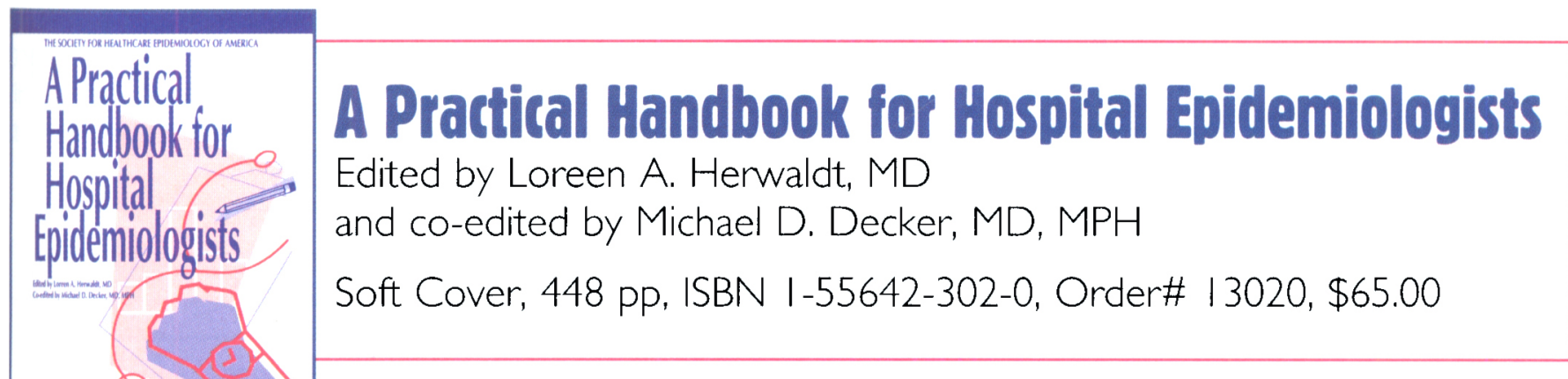

A Practical Handbook for Hospital Epidemiologists is the most complete source for practical advice on hospital epidemiology. It is intended to be a pragmatic guide that will assist both beginning and experienced epidemiologists in establishing and operating a successful hospital epidemiology program.

This handbook will supplement the various scientific references already available for hospital epidemiologists. It will provide practical information and advice regarding many aspects of operating a hospital epidemiology program and will help hospital epidemiologists improve their practices.

The Society for Healthcare Epidemiology of America (SHEA) has recruited the most recognized leaders in

\section{Contents}

Getting Started

Chapter I: An Introduction to Practical Hospital Epiderniology

Chapter 2: The Hospital Epidemiologist Practica ideas

Chapter 3: Educational Needs and Opportunities for the Hospital Epidemiologist

Chapter 4: Negotiating with the Administrationor How to Get Paid for Doing Hospital Epidemiology

Chapter 5: The Infection Control Committee

Chapter 6: Developing Policies and Guidelines

Chapter 7: Intramural and Extramural Communication

Chapter 8: Ethical Aspects of Infection Control

Surveillance and Analysis

Chapter 9: Basics of Surveilance-An Overview

Chapter 10: Hospital-Acquired Pneumonia:

Perspectives for the Healthcare Epidemiologist

Chapter II: Basics of Surgical Site Infection

Surveilance

Chapter 12: Surveillance for Infections Associated with Vascular Catheters

Chapter 13: Designing Survellance for Noninfectious Outcomes of Medical Care

Chapter 14: Outbreak Investigations

Chapter 15: Exposure Workups

Chapter 16: Isolation

Chapter 17: Basics of Stratifying for Severity of Ilness

Support Functions

Chapter 19: Microcomputers in Hospita Epidemiology

Chapter 20. The Computer-Based Patient Record The Role of the Hospital Epidemiologist

Chapter 21: Basic Microbiologic Support for Hospital Epidemiology

Chapter 22: Epidemiologic Typing Systems

Special Topics

Chapter 23: Epidemiologic Approaches to Quality Assessment

Chapter 24: Disinfection and Sterilization of Patient Care Items

Chapter 25: Controlling Use of Antimicrobial Agents

Chapter 26: Employee Heath and Infection Control

Chapter 27: Tuberculosis Control in Healthcare

Chapter 28: Infection Control Issues in Construction and Renovation

Chapter 29: Hospital Epidemiology in Smaller Hospitals

Chapter 30: Infection Control in Public Hospitals

Chapter 3I: Infection Control in Long-Term Care Facilities

Chapter 32: Infection Control in the Outpatient Setting

Chapter 33: OSHA Inspections

Chapter 34: Preparing for and Surviving a JCAHO Inspection the field to share their expertise. They will share successful strategies for handling specific situations. The authors cover many topics that every newcomer should know but are usually learned through experience. These topics include:

- Overarching goals and ethical principles you should adopt to guide your practice

-What you can do to educate yourself

- How to negotiate with the administration and communicate with colleagues

- How to develop policies and procedures

- How to develop a surveillance system

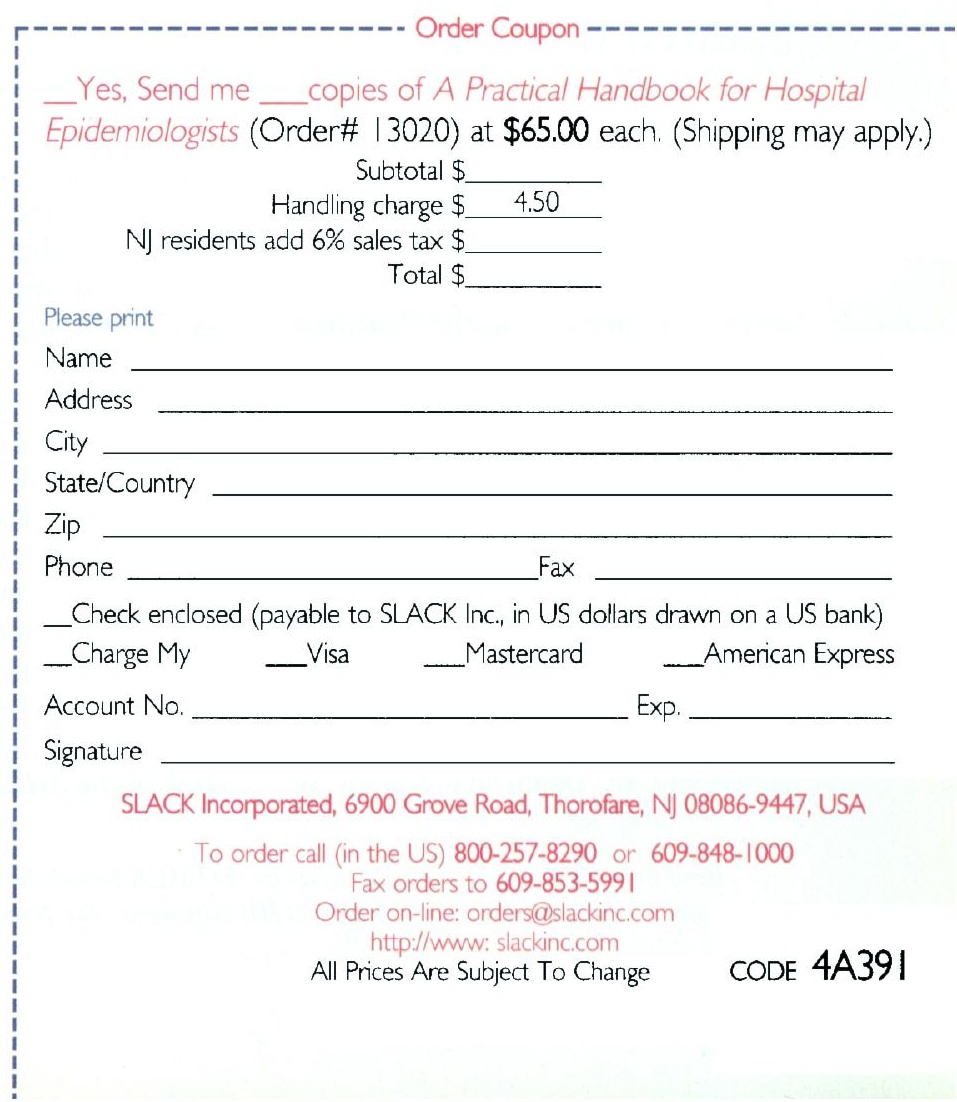

